\title{
Confronting, and Overcoming, Environmental Lead Poisoning Using Citizen Science
}

GABRIEL FILIPPELLI ${ }^{1}$, MARK TAYLOR $^{2}$, JANE A $^{2}$ ENTWISTLE $^{3}$, CYNTHIA ISLEY $^{2}$ AND JOHN T SHUKLE ${ }^{4}$

${ }^{1}$ Indiana University Purdue University Indianapolis

${ }^{2}$ Macquarie University

${ }^{3}$ Northumbria University

${ }^{4}$ Indiana University - Purdue University Indianapolis

Presenting Author: gfilippe@iupui.edu

Pollution from multiple sources causes significant disease and death worldwide. Some sources are legacy, such as heavy metals accumulated in soils, and some are current, such as particulate matter. Because the global burden of disease from pollution is so high, and so unequally distributed, it is important to identify legacy and current sources and to develop and implement effective techniques to reduce human exposure. One technique is through citizen-science, which is helping communities without adequate resources measure their own environments, and in this way gain agency in controlling local pollution exposure sources and/or alerting authorities to environmental hazards. This approach can yield a low cost, high access pollution sensing network at the scale of where people live, providing relevant personal environmental exposure data, engaging participants in the scientific process, and yielding valuable research results.

We have employed community-engaged research and citizen science, through the lens of lead poisoning, on a number of fronts. Several of us have developed and deployed large-scale garden soil programs to map the signature of lead contamination and to develop clear messaging to participants to mitigate hazards where needed. Additionally, we co-developed a global partnership to explore the indoor exposome (www.MapMyEnvironment.com) which is already yielding scientific insights into potential indoor risks from exposures. Through engagement with communities, particular low-income communities of color, we are attempting to eradicate environmental injustice and racism, enhance opportunities for training and youth development, and reach across the abyss that exists between universities and the very communities surrounding them. The future of GeoHealth will depend on building on these developments and others to protect a growing population from multiple pollution exposure risks.

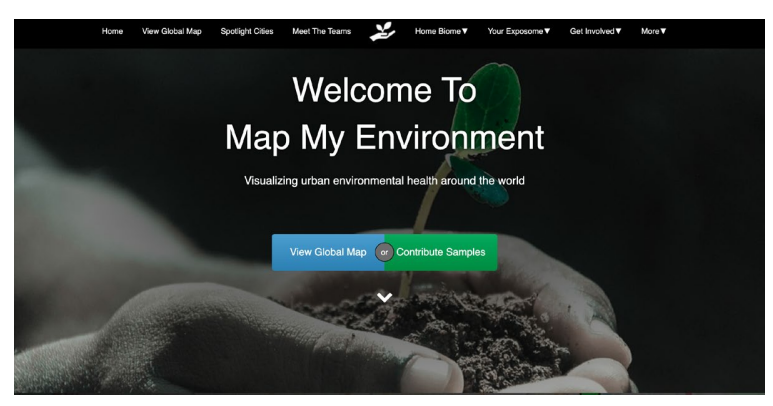

